\title{
A Featured Tuning of the Simulated Annealing Applied to the Open Shop Scheduling
}

\author{
Chaouqi Mohsine \\ OSIL Team LRI, ENSEM, \\ KM7, BP 8118 Route El Jadida \\ Casablanca, Morocco
}

\author{
Benhra Jamal \\ OSIL Team LRI, ENSEM, \\ KM7, BP 8118 Route El Jadida \\ Casablanca, Morocco
}

\author{
My Ali El Oualidi \\ OSIL Team LRI, ENSEM, \\ KM7, BP 8118 Route El Jadida \\ Casablanca, Morocco
}

\begin{abstract}
The present paper discusses the open shop scheduling problem using a manual tuning of a simulated annealing algorithm's parameters. A comparison has been done between Taillard's Benchmarks for 60 instances, 2 dispatching rules and 1296 variants of the SA algorithm obtained by changing the initial solution, the epoch length, and the steps' number, the initial temperature, the neighborhood and the cooling scheme.
\end{abstract}

The gotten results lead to some interesting conclusions for the best choice of the parameters.

\section{General Terms}

Algorithm, parameters, tuning.

\section{Keywords}

Scheduling, Simulated annealing, epoch length, neighborhood, cooling scheme, tuning, and open shop.

\section{INTRODUCTION}

Shop scheduling involves the processing of a set of jobs on a set of machines by defining the time intervals in which the operations have to be processed. [1]

There are three basic types of shops: a flow-shop (each job is characterized by the same technological route), a job-shop (each job has a specific route) and an open shop (no technological route is imposed on the jobs). [2]

However there exist different objectives in scheduling optimization. The most known objective is the makespan Cmax minimization which is the time's span required to process all the jobs, i.e. the time from the beginning of the first operation until the end of the last operation. The second one is to minimize the flowtime, denoted by $\sum \mathrm{Cj}$, which is the completion times' sum of all the jobs. Other objectives are the tardiness's minimization, the number of tardy jobs, etc.

In this article a manual tuning of the simulated annealing's parameters is made and the given results were compared with Taillard's benchmarks for 60 instances where $n=m \in\{4,5,7$, $10,15,20\}$.

The present paper is organized as follow: Section 2 contains a short reminder of the different works found on the open shop scheduling optimization. Section 3 sets out the algorithm used and the tuning made. In section 4 the gotten results are presented and their interpretation. Finally this article ends with a conclusion and some perspectives.

\section{LITERATURE REVIEW}

As mentioned before the open shop is one type of the three basic shops where a set of $n$ jobs $J_{1}, J_{2}, \ldots, J_{n}$ has to be processed on a set of $\mathrm{m}$ machines $\mathrm{M}_{1}, \mathrm{M}_{2}, \ldots, \mathrm{M}_{\mathrm{m}}$ with an arbitrary order. The operation $(i, j)$ means the processing of job $\mathrm{J}_{\mathrm{i}}$ on machine $\mathrm{M}_{\mathrm{j}}$.

One can find this kind of shop in a large aircraft garage with specialized work centers, in automobile repair, quality control centers, semiconductor manufacturing, teacher-class assignments, examination scheduling, and satellite communications as described in [3], [4] and [5].

There exist many works involving the open shop scheduling problem. One of them is that of Liaw [6] who used simulated annealing in case of minimizing makespan on a nonpremptive open shop. He proposed a neighborhood search. His algorithm was tested on randomly generated problems, benchmark problems. He got a good results but spent a lot of time to reach them (up to 3.5 hours per single run for an instance with $\mathrm{n}=\mathrm{m}=30$ ).

The same author, proposed in [7] a hybrid genetic algorithm (HGA) to resolove the open shop scheduling problem. The hybrid algorithm incorporates a local improvement procedure based on tabu search (TS) into a basic GA. The algorithm developed has been tested on randomly generated instances and on the benchmarks sets by Taillard [8] and Brucker et al. [9]. It has been found that this HGA outperformed other existing algorithms from the literature, and some benchmark instances have been solved to optimality for the first time.

Michael Andresen et al.[3] considered the problem of scheduling $\mathrm{n}$ jobs on $\mathrm{m}$ machines in an Open Shop environment with the minimization of total weighted tardiness as a goal. The main goal of their study was to find out which parameters have a strong influence and which have a smaller influence on the selection of an appropriate simulated annealing algorithm.

Fang et al. [10] suggested an algorithm which combines a GA with heuristic rules for the schedule construction. The algorithm has been tested on the benchmark instances from [8] using ten runs for each instance. By their tests, they discovered one new best known solution for a problem with 7 machines and 7 jobs and a problem with 10 machines and 10 jobs instance.

Rui Zhang et al.[11] executed a simulated annealing algorithm based on bottleneck jobs on an Open Shop scheduling problem. Their study attempt to minimize the total weighted tardiness.

Recently Bai et al [12] used a heuristic called general dense scheduling to solve the static and dynamic versions of the flexible open shop scheduling problem. The heuristic proposed brings forth some interesting results.

Naderi et al [13] studied the scheduling open shop problem with no intermediate buffer, called no-wait open shops under makespan minimization. They develop three mathematical 
models and propose metaheuristics based on genetic and variable neighborhood search algorithms. The results they got show that the models and metaheuristics are effective to deal with the no-wait open shop problems.

The simulated annealing algorithm proposed in this article was used by Chaouqi et al [14] in hybridization within the intuitive heuristic to perform a joint scheduling of production and maintenance in the job shop problem. On the same topic the authors used the Johnson's algorithm combined with a genetic algorithm and the intuitive heuristic to optimize three objectives of the flow shop problem. [15]

\section{SIMULATED ANNEALING AND PARAMETERS TUNING}

In the years 80 three researchers from IBM company $-\mathrm{S}$. Kirkpatrick, C. D. Gelatt and M.P. Vecchi have proposed and published a new iterative method called Simulated annealing Kirkpatrick et al.[16], which avoid the local minimum. Since that discovery, this method has been tested in different fields like the design of the electronic circuits, the image processing etc.

Simulated annealing is inspired from a metallurgic process called annealing. The annealing process is a heat treatment that alters the physical and sometimes chemical properties of a material to increase its ductility and reduce its hardness, making it more workable. It involves heating a material to above its recrystallization temperature, maintaining a suitable temperature, and then cooling [17]. This strategy leads to a crystallized solid state, which is a stable state, corresponding to an absolute minimum of energy.

Similarly to the real process simulated annealing involves two steps; heating and cooling. In the first step a control parameter called temperature is introduced which must lead the system to an optimal state. In the second one this temperature is reduced during certain epochs within a cooling parameter till the end of the period then it will be reinitialized.

Instead of Hill Climbing method, the simulated annealing accepts a worse solution with a certain probability. This one depends on the decreasing temperature so a local optimum is avoided.

In this paper where the objective is the minimization of the makespan, the algorithm is as follow [18]:

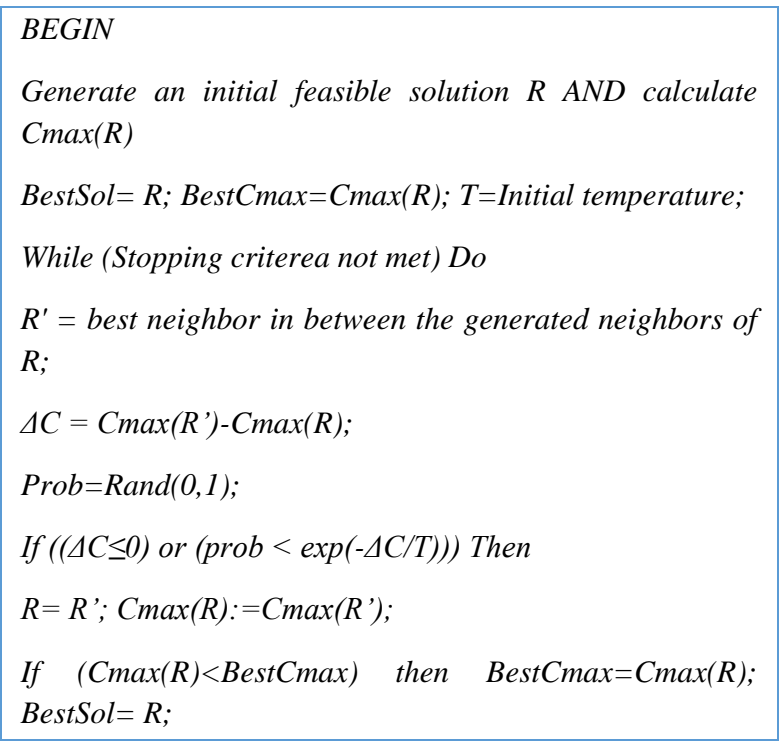

End if
Update T according to the chosen cooling scheme;
End if
End while
END

Figure 1: Simulated algorithm applied to open shop problem with makespan minimization as an objective

Where $\mathrm{R}$ is the rank matrix, Cmax is the makespan and neighbor is the new solution generated according to the chosen neighborhood.

\subsection{Rank matrix}

The rank matrix $R=\left(r_{i j}\right)$ describes a sequence graph $G(M O$, $\mathrm{JO})$ which is a feasible combination of machine orders and job orders. The rank $r_{i j}$ is the maximal number of operations on a longest path ending in operation $(i, j)[2]$.

Let the case $n=m=3$ where the job orders are described by the matrix JO and the machine orders are described by the matrix MO.

$$
M O=\left(\begin{array}{lll}
3 & 2 & 1 \\
1 & 2 & 3 \\
2 & 1 & 3
\end{array}\right) \quad J O=\left(\begin{array}{lll}
3 & 1 & 2 \\
3 & 2 & 1 \\
1 & 2 & 3
\end{array}\right)
$$

The graphs related to these two matrices are shown in figure 2:

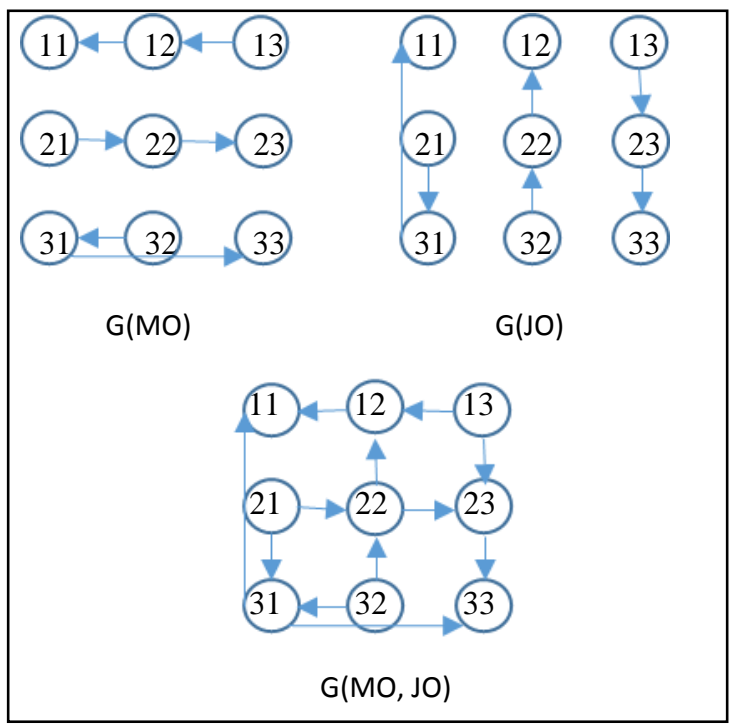

Figure 2: G(MO), G(JO) and G(MO, JO)

In the same figure the graph $\mathrm{G}(\mathrm{MO}, \mathrm{JO})$ is given which is the combination of the two graphs.

\subsection{Neighborhoods}

In this paper nine neighborhoods are used. Here a brief description of them is given.

The first one is the PI neighborhood which stands for pairwise interchange where two arbitrary operations are interchanged. The second one is the API neighborhood. This neighborhood consists on interchanging two adjacent operations on a rank matrix to get a new neighbor, i.e an operation $(i, j)$ is randomly selected and then it is interchanged with the predecessor or 
successor operation on machine $\mathrm{M}_{\mathrm{j}}$ or of job $\mathrm{J}_{\mathrm{i}}$. If the pairwise interchange leads to a feasible schedule, it is accepted as a neighbor, otherwise another second operation is chosen to perform a new adjacent pairwise interchange. The neighborhood k_API is the same as the API with the exception of generating $\mathrm{k}$ neighbors. When generating a neighbor, the interchanges' number of two adjacent operations or on a machine of a job is randomly chosen. However in CR_API (resp. BL_API) neighborhood a Cmax critical operation (resp. block-end-operation) is interchanged with a directly adjacent operation.

In the neighborhood SHIFT one operation is changed in the relative order of operations, i.e. one operation is shifted left or right in the job order on one machine or in the machine order of one job. The operation is chosen randomly. Then another operation belonging to the same job or to be processed on the same machine is selected, e.g. let (i, k) be the other chosen operation. If the rank aik is smaller than $\mathrm{a}_{\mathrm{ij}}$, the rank $\mathrm{a}_{\mathrm{ij}}$ is modified such that operation (i, j) appears immediately before (i, k) (it corresponds to a right shift of machine $M_{j}$ in the machine order of job $\mathrm{J}_{\mathrm{i}}$ ). If the generated solution is infeasible, two other operations will be chosen randomly for performing a shift. In the BL_SHIFT a Cmax critical block-end-operation is shifted in the sequence. However the CR_SHIFT neighborhood which is a sub-neighborhood of the SHIFT neighborhood generates new neighbors from this one where the old ones satisfy a necessary condition for an improvement of the makespan, namely a critical path in the starting solution is destroyed, and there does not exist a path in the graph describing the generated neighbor which contains the same vertices as this critical path of the current starting solution.

Finally in this paper two other neighborhoods are used: the 3_CR where a Cmax critical operation is interchanged with a directly adjacent operation.[3] [19]

\section{RESULTS AND DISCUSSION}

In this section, computational results are presented for the simulated annealing. The parameters used in this study are:

Steps $\epsilon\{5000,10000,15000,20000\}$, Epoch_length $\epsilon\{100$, $200,300\}$, initial_temperature $\epsilon\{10,20,30\}$, cool_scheme $\epsilon$ \{GEOMETRIC, LUNDYANDMEES\}, initial_solution $\epsilon$ $\{$ SPT, LPT $\}$ neighborhood $\epsilon$ PPI, API, k_API, CR_API, BL_API, SHIFT, BL_SHIFT, CR_SHIFT, 3_CR,$\quad \mathrm{k}=3$, number of neighbors $=1$, cool_paramter $=0.0005$. Thus the number of variants is $4 * 3 * 3 * 2 * 2 * 9=1296$ variants.

The time limit is 10 seconds fixed as a stopping criterion.

\subsection{Case $^{\circ} 1$ : $\mathrm{Cmax}=$ Optimum}

Here we describe the frequencies of variants which solved a certain number of problems out of the sixty instances given by Taillard, i.e. the final makespan calculated for these variants has reached the optimums of the corresponding problem. The table $n^{\circ} 1$ presents the gotten results in this first case.

Table 1. Number of variants by the number of solutions where $\mathbf{C m a x}=\mathbf{O p t}$

\begin{tabular}{|c|c|}
\hline Variants' number & Solutions' number \\
\hline 831 & 0 \\
\hline 196 & 1 \\
\hline 97 & 2 \\
\hline 55 & 3 \\
\hline
\end{tabular}

\begin{tabular}{|c|c|}
\hline 50 & 4 \\
\hline 26 & 5 \\
\hline 18 & 6 \\
\hline 13 & 7 \\
\hline 6 & 8 \\
\hline 3 & 9 \\
\hline 1 & 10 \\
\hline
\end{tabular}

One can see in the figure $n^{\circ} 3$, most of variants do not generate an optimal solution for each instance from the sixty instances which is undesirable, i.e. the tuning suggested is not as good as expected. Only one configuration reached optimum solution for 10 out of 60 . In this one the epoch length is 100 , the number of steps is equal to 20000 , the initial temperature is 10 , the cooling parameter is 0.0005 , the neighborhood is CR_SHIFT the initial solution is LPT and the cooling scheme is GEOMETRIC. This variant will be denoted by SA1.

The second good result, which is 9 optimums over 60 , is given by three configurations: SA2 $=$ (epoch_length $=200$, steps $=20000, \quad$ t_start $=10$, cool_parameter $=0.0005$, neighborhood $=$ CR_SHIFT, cool_scheme $=$ GEOMETRIC, init_sol $=$ LPT $),$ SA3 $=(100,15000,10,0.0005$, CR_SHIFT, GEOMETRIC, LPT) and SA4 $=(200,20000,10,0.0005$, CR_SHIFT, LUNDYANDMEES).

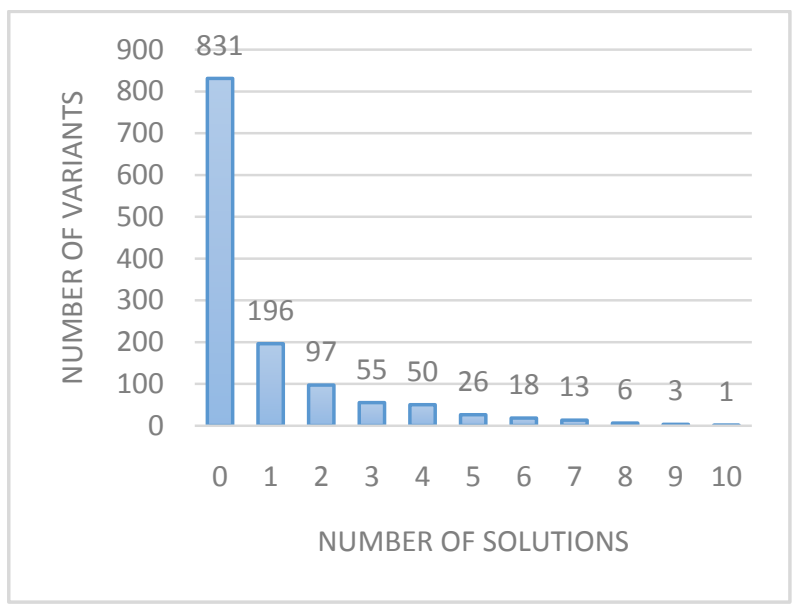

Figure 3: Number of variants by the number of solutions where $\mathbf{C m a x}=$ Opt

To get more information from the generated data a deep analyze of them is made by studying another case; where the ratio Optimum/Cmax is greater than or equal to 0.99 .

\subsection{Case $^{\circ}$ 2: Optimum/Cmax $>=0.99$}

In this second case a new distribution is given which differs from the previous one. One can observe in figure 4 a decreasing graph from 0 to 4 solutions then a new pic and again a decreasing graph from 5 to 18 .

The maximal number of solutions with Opt/Cmax $>=0.99$ is 18 out of 60 reached by one variant: SA1, followed by 16 out of 60 obtained for these two configurations SA2 and SA3.

Here one can observe that the three variants SA1, SA2 and SA3 performed well in either cases. 


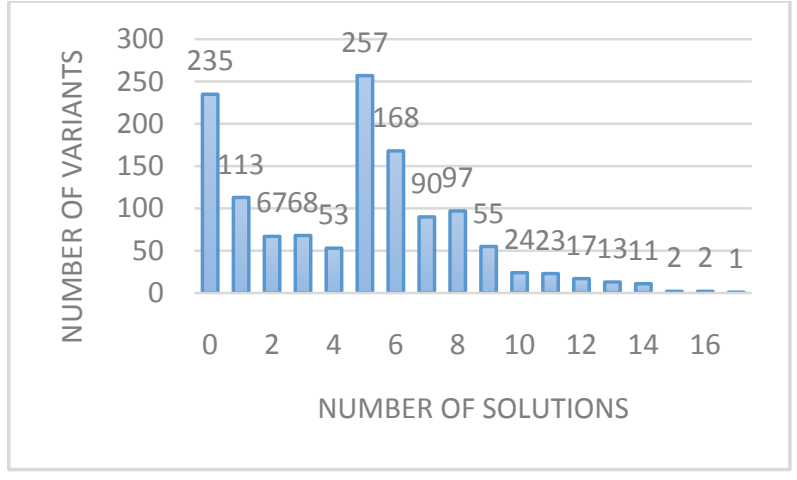

Figure 4: Number of variants by the number of solutions where Optimum/Cmax $>=0.99$

We grouped all the results of SA1, SA2 and SA3 applied to the Taillard's problem, with those given by SPT and LPT rules in table $\mathrm{n}^{\circ} 2$. The abbreviation LB means the lower bound and Opt stands for the optimum found in the literature.

Table 2. Results for the benchmark problems from Taillard

\begin{tabular}{|c|c|c|c|c|c|c|c|}
\hline$(n x m) \_i$ & $L B$ & Opt & $S P T$ & $\begin{array}{c}\boldsymbol{L P} \\
T\end{array}$ & $S A 1$ & $\mathrm{SA} 2$ & SA3 \\
\hline$(4 x 4) \_1$ & 186 & 193 & 228 & 219 & 193 & 193 & 193 \\
\hline$(4 x 4) \_2$ & 229 & 236 & 276 & 256 & 236 & 239 & 236 \\
\hline$(4 x 4) \_3$ & 262 & 271 & 304 & 299 & 271 & 271 & 272 \\
\hline$(4 x 4) \_4$ & 245 & 250 & 307 & 260 & 250 & 250 & 250 \\
\hline$(4 x 4) \_5$ & 287 & 295 & 348 & 317 & 295 & 303 & 295 \\
\hline$(4 x 4) \_6$ & 185 & 189 & 225 & 239 & 189 & 189 & 189 \\
\hline$(4 x 4) \_7$ & 197 & 201 & 247 & 218 & 201 & 201 & 201 \\
\hline$(4 x 4) \_8$ & 212 & 217 & 233 & 248 & 217 & 217 & 217 \\
\hline$(4 x 4) \_9$ & 258 & 261 & 282 & 282 & 261 & 261 & 261 \\
\hline$(4 x 4) \_10$ & 213 & 217 & 235 & 225 & 217 & 217 & 217 \\
\hline$(5 \times 5) \_11$ & 295 & 300 & 333 & 344 & 301 & 303 & 305 \\
\hline$(5 \times 5) \_12$ & 255 & 262 & 297 & 297 & 263 & 270 & 263 \\
\hline$(5 \times 5) \_13$ & 321 & 323 & 404 & 364 & 336 & 338 & 337 \\
\hline$(5 \times 5) \_14$ & 306 & 310 & 317 & 369 & 322 & 319 & 316 \\
\hline$(5 \times 5) \_15$ & 321 & 326 & 392 & 358 & 339 & 339 & 339 \\
\hline$(5 \times 5) \_16$ & 307 & 312 & 353 & 360 & 322 & 327 & 324 \\
\hline$(5 \times 5) \_17$ & 298 & 303 & 340 & 357 & 314 & 308 & 307 \\
\hline$(5 \times 5) \_18$ & 292 & 300 & 369 & 343 & 312 & 301 & 314 \\
\hline$(5 \times 5) \_19$ & 349 & 353 & 372 & 418 & 367 & 362 & 357 \\
\hline$(5 \times 5) \_20$ & 321 & 326 & 375 & 371 & 332 & 326 & 332 \\
\hline (7x7)_21 & 435 & 435 & 507 & 465 & 459 & 453 & 455 \\
\hline
\end{tabular}

\begin{tabular}{|c|c|c|c|c|c|c|c|}
\hline$(7 x 7) \_22$ & 443 & 443 & 485 & 526 & 459 & 470 & 459 \\
\hline$(7 x 7) \_23$ & 468 & 468 & 538 & 527 & 500 & 498 & 500 \\
\hline (7x7)_24 & 463 & 463 & 492 & 527 & 494 & 472 & 474 \\
\hline (7x7)_25 & 416 & 416 & 461 & 443 & 434 & 429 & 434 \\
\hline (7x7)_26 & 451 & 451 & 518 & 494 & 469 & 490 & 469 \\
\hline$(7 x 7) \_27$ & 422 & 422 & 464 & 451 & 445 & 436 & 445 \\
\hline (7x7)_28 & 424 & 424 & 482 & 494 & 442 & 447 & 442 \\
\hline$(7 x 7) \_29$ & 458 & 458 & 520 & 488 & 472 & 472 & 472 \\
\hline (7x7)_30 & 398 & 398 & 435 & 445 & 416 & 429 & 407 \\
\hline $\begin{array}{c}(10 \times 10)+3 \\
1\end{array}$ & 637 & 637 & 685 & 661 & 661 & 661 & 661 \\
\hline $\begin{array}{c}(10 \times 10) \_3 \\
2\end{array}$ & 588 & 588 & 658 & 643 & 606 & 607 & 633 \\
\hline $\begin{array}{c}(10 \times 10)+3 \\
3\end{array}$ & 598 & 598 & 679 & 672 & 650 & 662 & 647 \\
\hline $\begin{array}{c}(10 \times 10) \_3 \\
4\end{array}$ & 577 & 577 & 632 & 591 & 584 & 591 & 584 \\
\hline $\begin{array}{c}(10 \times 10) \_3 \\
5\end{array}$ & 640 & 640 & 693 & 701 & 688 & 677 & 686 \\
\hline $\begin{array}{c}(10 \times 10) \_3 \\
6\end{array}$ & 538 & 538 & 559 & 556 & 554 & 555 & 555 \\
\hline $\begin{array}{c}(10 \times 10) \_3 \\
7\end{array}$ & 616 & 616 & 672 & 637 & 635 & 637 & 637 \\
\hline $\begin{array}{c}(10 \times 10){ }^{3} 3 \\
8\end{array}$ & 595 & 595 & 651 & 686 & 623 & 644 & 630 \\
\hline $\begin{array}{c}(10 \times 10) \\
9\end{array}$ & 595 & 595 & 655 & 621 & 621 & 621 & 621 \\
\hline $\begin{array}{c}(10 \times 10)+4 \\
0\end{array}$ & 596 & 596 & 633 & 636 & 630 & 621 & 634 \\
\hline $\begin{array}{c}(15 \times 15) \_4 \\
1\end{array}$ & 937 & 937 & 987 & 972 & 970 & 972 & 972 \\
\hline $\begin{array}{c}(15 \times 15) \_4 \\
2\end{array}$ & 918 & 918 & 937 & 972 & 971 & 971 & 969 \\
\hline $\begin{array}{c}(15 \times 15) \_4 \\
3\end{array}$ & 871 & 871 & 891 & 878 & 876 & 878 & 878 \\
\hline $\begin{array}{c}(15 \times 15) \\
4\end{array}$ & 934 & 934 & 975 & 965 & 939 & 949 & 964 \\
\hline $\begin{array}{c}(15 \times 15) \_4 \\
5\end{array}$ & 946 & 946 & 959 & 999 & 990 & 990 & 990 \\
\hline $\begin{array}{c}(15 \times 15) \_4 \\
6\end{array}$ & 933 & 933 & 981 & 952 & 951 & 950 & 952 \\
\hline$(15 \times 15) \_4$ & 891 & 891 & 919 & 955 & 950 & 954 & 954 \\
\hline
\end{tabular}




\begin{tabular}{|c|c|c|c|c|c|c|c|}
\hline $\begin{array}{c}(15 \times 15) \_4 \\
8\end{array}$ & 893 & 893 & 928 & 929 & 920 & 919 & 920 \\
\hline $\begin{array}{c}(15 \times 15) \_4 \\
9\end{array}$ & 899 & 899 & 990 & 927 & 927 & 927 & 927 \\
\hline $\begin{array}{c}(15 \times 15) \_5 \\
0\end{array}$ & 902 & 902 & 922 & 943 & 942 & 941 & 943 \\
\hline (20x20)_5 & 115 & 115 & 119 & 120 & 119 & 119 & 120 \\
\hline 1 & 5 & 5 & 4 & 0 & 6 & 9 & 0 \\
\hline$(20 \times 20) \_5$ & 124 & 124 & 129 & 129 & 128 & 129 & 129 \\
\hline 2 & 1 & 1 & 6 & 6 & 9 & 6 & 6 \\
\hline (20x20)_5 & 125 & 125 & 130 & 125 & 125 & 125 & 125 \\
\hline 3 & 7 & 7 & 4 & 8 & 8 & 8 & 8 \\
\hline (20x20)_5 & 124 & 124 & 131 & 127 & 127 & 126 & 127 \\
\hline 4 & 8 & 8 & 2 & 4 & 4 & 4 & 4 \\
\hline (20x20)_5 & 125 & 125 & 127 & 126 & 126 & 126 & 126 \\
\hline 5 & 6 & 6 & 7 & 2 & 0 & 2 & 0 \\
\hline (20x20)_5 & 120 & 120 & 121 & 121 & 121 & 121 & 121 \\
\hline 6 & 4 & 4 & 9 & 5 & 5 & 5 & 3 \\
\hline$(20 \times 20) \_5$ & 129 & 129 & 140 & 131 & 131 & 131 & 131 \\
\hline 7 & 4 & 4 & 7 & 7 & 3 & 2 & 7 \\
\hline (20x20)_5 & 116 & 116 & 120 & 121 & 121 & 121 & 121 \\
\hline 8 & 9 & 9 & 5 & 6 & 5 & 5 & 5 \\
\hline (20x20)_5 & 128 & 128 & 130 & 129 & 129 & 129 & 129 \\
\hline 9 & 9 & 9 & 6 & 3 & 2 & 2 & 2 \\
\hline$(20 \times 20) \_6$ & 124 & 124 & 127 & 126 & 125 & 125 & 126 \\
\hline 0 & 1 & 1 & 2 & 5 & 9 & 9 & 5 \\
\hline
\end{tabular}

One can notice that the gotten results using the three variants SA1, SA2 and SA3 for the sixty instances are very close to the optimums found in the literature, and better than the two dispatching rules.

The worst value of the makespan given by the SA1 compared to the optimum value was 650 for the 33th instance where $\mathrm{n}=\mathrm{m}=10$, and the optimum is equal to 598 . For this value the ratio $\mathrm{Opt} / \mathrm{Cmax}$ is $92 \%$ which is acceptable.

Again the worst obtained values for the SA2 and SA3 are respectively 662 and 647 also obtained for the 33th instance. Thus the worst values for the ratio Opt/Cmax are $90 \%$ and $92 \%$ which still acceptable.

In addition to the ten first instances where $\mathrm{Opt}=\mathrm{Cmax}$ for the SA1 variant, there are five other instances where the ratio Opt/Cmax is approximatively equal to $100 \%$. Those instances are $(5 \times 5) \_11, \quad(5 \times 5) \_12, \quad(20 \times 20) \_53, \quad(20 \times 20) \_55$ and (20x20)_59.

\section{CONCLUSION}

In this paper a comparison has been done between Taillard's Benchmarks for 60 instances in the open shop problem, the SPT and the LPT dispatching rules and 1296 variants of the simulated annealing algorithm obtained by changing the initial solution, the epoch length, the number of steps, the initial temperature, the neighborhood and the cooling scheme.

The gotten results are interesting in few cases. This is maybe due to the time limit set as a stopping criterion or it is due to the chosen simulated annealing algorithm implementation which needs some improvements. However some variants yield great results.

As a perspective of future studies, one can use some other tools like neural networks or Bayesian networks and a hybridization in between different algorithms to get a better tuning of the SA parameters then try new tests to get an optimum makespan for all instances.

\section{REFERENCES}

[1] M. L. Pinedo, Scheduling, vol. 1. Boston, MA: Springer US, 2012

[2] F. Werner, "Genetic algorithms for shop scheduling problems: A survey," Preprint, 2011.

[3] O. Magdeburg, F. Mathematik, M. Andresen, H. Bräsel, M. Plauschin, and F. Werner, "Using Simulated Annealing for Open Shop Scheduling with Sum Criteria," pp. 1-26, 2008.

[4] C. Prins, "An Overview of Scheduling Problems Arising in Satellite Communications," J. Oper. Res. Soc., vol. 45, no. 6, p. 611, Jun. 1994.

[5] C. Y. Liu and R. L. Bulfin, "Scheduling ordered open shops," Comput. Oper. Res., vol. 14, no. 3, pp. 257-264, Jan. 1987.

[6] C. F. Liaw, "Applying simulated annealing to the open shop scheduling problem," IIE Trans. (Institute Ind. Eng., vol. 31, no. 5, pp. 457-465, 1999.

[7] C.-F. Liaw, "A hybrid genetic algorithm for the open shop scheduling problem," Eur. J. Oper. Res., vol. 124, no. 1, pp. 28-42, Jul. 2000.

[8] Taillard E., "Benchmarks for basic scheduling problems," Eur. J. Oper. Res., vol. 64, pp. 1-17, 1993.

[9] P. Brucker, J. Hurink, B. Jurisch, and B. Wöstmann, "A branch \& bound algorithm for the open-shop problem," Discret. Appl. Math., vol. 76, no. 1-3, pp. 43-59, Jun. 1997.

[10] H. Fang and P. Ross, "A Promising Hybrid GA/Heuristic Approach for Open-Shop Scheduling Problems In Proceedings of the 11th European Conference on Arti cial Intelligence, John Wiley and Sons, 1994, pages 590\{594.," no. 699, 1994.

[11] R. Zhang and C. Wu, "An Immune Genetic Algorithm Based on Bottleneck Jobs for the Job Shop Scheduling," no. 1, pp. 147-157, 2008.

[12] D. Bai, Z.-H. Zhang, and Q. Zhang, "Flexible open shop scheduling problem to minimize makespan," Comput. Oper. Res., vol. 67, pp. 207-215, Mar. 2016.

[13] B. Naderi and M. Zandieh, "Modeling and scheduling no-wait open shop problems," Int. J. Prod. Econ., vol. 158, pp. 256-266, Dec. 2014.

[14] M. Chaouqi and J. Benhra, "Recuit simulé hybride pour un ordonnancement conjoint de la production et de la maintenance dans un atelier job-shop," Int. Work. Theory Appl. Logist. Transp. TALT15, 2015. 
[15] M. Chaouqi, J. Benhra, and A. Zakari, "Agile Approach for Joint Scheduling of Production and Maintenance in Flow Shop," Int. J. Comput. Appl., vol. 59, no. 11, pp. 29-36, Dec. 2012.

[16] M. P. V. S. Kirkpatrick C. D. Gelatt, "Optimization by Simulated Annealing," Science (80-. )., vol. 220, no. 4598, pp. 671-680, 1983.

[17] "Annealing - Wikipedia, the free encyclopedia." [Online].

Available:

https://en.wikipedia.org/wiki/Annealing. [Accessed: 19-
Mar-2016].

[18] K. Hasani, S. A. Kravchenko, and F. Werner, "Minimizing the makespan for the two-machine scheduling problem with a single server: Two algorithms for very large instances," Eng. Optim., vol. 48, no. 1, pp. 173-183, 2016.

[19] M. Andresen, F. Engelhardt, and F. Werner, "LiSA - A Library of Scheduling Algorithms Handbook for Version 3.0," 2010 . 